\title{
Paediatric cardiology
}

GW23-e1076 THE CLINICAL SIGNIFICANCE OF SERUM LEVELS OF CK-MB AND CTN-I COMBINED WITH CORRECTED OT DISPERSION TO EVALUATE THE SEVERITY OF MYOCARDIAL INJURY AFTER ASPHYXIA IN NEONATES

doi:10.1136/heartjnl-2012-302920s.1

\author{
${ }^{1}$ Fang Xiao-yi, ${ }^{2}$ Li Yu-guang, ${ }^{1}$ Weng Li-Jian, ${ }^{1}$ Yang Si-Lan, ${ }^{1}$ Lin Ni-Yang. ${ }^{1}$ Department of \\ Neonatology, First Affiliated Hospital of Shantou University Medical College; \\ ${ }^{2}$ Department of Cardiology, First Affiliated Hospital of Shantou University Medical \\ College
}

Objectives Hypoxic ischaemic myocardial injury is one of the serious complications of asphyxia in neonates, which may cause systolic dysfunction, arrhythmias, heart failure and even death. Early and accurate diagnosis may direct the appropriate treatments. The aim of this study was to investigate the significance of serum level of creatine kinase- $\mathrm{MB}$ (CK-MB) and cardiac troponin-I (cTnI) combined with corrected OT dispersion (OTcD) to evaluate the severity of post-asphyxial myocardial injury in neonates.

Methods We enrolled 52 neonates in asphyxia group (38 in mild asphyxia group and 14 in severe asphyxia group) and 30 neonates in control group. There were no significant differences among the three groups in terms of gestational age, birth weight, gender, and age. Serum levels of CK-MB and cTnI were detected by enzyme linked immunosorbent assay (ELISA) and immunodepression and OTcD were calculated by 12-lead electrocardiagrams (ECG) at the 1st-3rd day after birth in asphyxia and control groups and 7th10th day after birth in asphyxia group respectively. Data were statistically analysed using SPSS 13.0 software.

Results Before treatment, the serum levels of CK-MB were 201.0 $\pm 102.80,281.21 \pm 163.78$ and $22.0 \pm 6.69(\mathrm{U} / \mathrm{L}), \mathrm{cTnI}$ were 1.13 $\pm 0.40,2.67 \pm 0.60$ and $0.30 \pm 0.17(\mathrm{ng} / \mathrm{ml})$ and $\mathrm{QTcD}$ were 62.22 \pm 30.37 , 76.24 \pm 27.72 and $38.44 \pm 21.76$ (ms) in mild asphyxia, severe asphyxia and control group respectively $(p<0.01)$. The serum levels of CK-MB and $\mathrm{CTnI}$ and $\mathrm{QTcD}$ values in mild and severe asphyxia group were higher than those in control group. After treatment, the serum levels of CK-MB were $38.74 \pm 18.08$ and $52.93 \pm 40.61(\mathrm{U} / \mathrm{L}), \mathrm{cTn} I$ were $0.37 \pm 0.19$ and $0.98 \pm 0.22(\mathrm{ng} /$ $\mathrm{ml}$ ) and $\mathrm{OTcD}$ were $41.82 \pm 20.42$ and $54.40 \pm 31.43(\mathrm{~ms})$ in mild and severe asphyxia group respectively. Compared with those values before treatment, the CK-MB and cTnI levels and OTcD were all decreased in two asphyxia groups after treatment. Before or after the treatment, the differences of CK-MB levels and $\mathrm{OTCD}$ values between mild and severe asphyxia group were not significant $(p>0.05)$. But the cTnI levels were significantly different between two asphyxia groups before or after treatment $(p<0.01)$. The CK-MB level, cTnI level and OTcD value were negatively correlated with the Apgar score at the first minute after birth $(\mathrm{r}=$ $-0.742,-0.943$ and -0.58 respectively with $p<0.01)$. The sensitivity of CK-MB, cTnI and OTcD was $100 \%, 96.15 \%$ and $15.38 \%$ respectively, while the specificity was $66.67 \%, 86.67 \%$ and $93.33 \%$ respectively.

Conclusions Serum levels of CK-MB and cTnI and OTcD values were increased in neonates with asphyxia, which may indicate the myocardial injury. cTnI is a better index to evaluate the severity of myocardial injury after asphyxia than CK-MB and OTcD. CK-MB level has high sensitivity while $\mathrm{OTCD}$ value has high specificity. CK-MB and cTnI level combined with OTcD might increased the diagnostic accuracy of myocardial injury after asphyxia in neonates. 